\title{
Separable Hilbert space in Loop Quantum Gravity
}

\author{
Winston Fairbairn ${ }^{a *}$, Carlo Rovelli ${ }^{a, b \dagger}$ \\ ${ }^{a}$ Centre de Physique Théorique de Luminy, Case 907, F-13288 Marseille, EU \\ ${ }^{b}$ Dipartimento di Fisica, Università "La Sapienza", P Moro 2, I-00185 Roma, EU
}

October 25, 2018

\begin{abstract}
We study the separability of the state space of loop quantum gravity. In the standard construction, the kinematical Hilbert space of the diffeomorphism-invariant states is nonseparable. This is a consequence of the fact that the knot-space of the equivalence classes of graphs under diffeomorphisms is noncountable. However, the continuous moduli labeling these classes do not appear to affect the physics of the theory. We investigate the possibility that these moduli could be only the consequence of a poor choice in the fine-tuning of the mathematical setting. We show that by simply choosing a minor extension of the functional class of the classical fields and coordinates, the moduli disappear, the knot classes become countable, and the kinematical Hilbert space of loop quantum gravity becomes separable.
\end{abstract}

\section{Introduction}

Loop quantum gravity (LQG) is a background-independent quantization of classical general relativity which yields a discrete, combinatorial picture of Planck-scale quantum geometry. ${ }^{1}$ Quantum space turns out to be described in terms of a basis of abstract spin-network states, or $s$-knot states, labeled by discrete quantum numbers. However, the picture is not truly entirely discrete. If the nodes of the $s$-knots have sufficiently high valence (that is, when a sufficiently high number of links meet), $s$-knots are labelled also by certain continuous moduli parameters. The existence of these moduli was pointed out in [11] and their structure studied in [6]. Below we give an example of one of these moduli explicitly. These moduli are puzzling. They are virtually undetectable by the operators that represent physical measurements, as well as by the hamiltonian operator that governs the dynamics, and therefore they do not appear to play any significant role in the theory. Still, they spoil the discreetness of the picture and they change the structure of the space of the diffeomorphism-invariant states, $\mathcal{H}_{\text {diff }}$, rather drastically, making it nonseparable. ${ }^{2}$

Nonseparability (absence of a countable basis) is generally regarded as pathological in quantum field theory (QFT). A classic discussion on this issue is in [13]. As pointed out in [14, the nonseparability of $\mathcal{H}_{\text {diff }}$ is not necessarily unacceptable, because $\mathcal{H}_{\text {diff }}$ is a kinematical space that must still be reduced by the hamiltonian constraint equation. But it is nevertheless disturbing. Do we have to interpret it as an indication of a difficulty of the background-independent loop quantization?

In this paper, motivated by the fact that the moduli do not appear to have any physical significance, we consider the possibility that they are indeed spurious. We study the possibility

\footnotetext{
*fairbairn@cpt.univ-mrs.fr

${ }^{\dagger}$ rovelli@cpt.univ-mrs.fr

${ }^{1}$ For an introduction and references, see for instance 9 .

${ }^{2}$ The space $\mathcal{H}_{\text {diff }}$ is denoted $\mathcal{K}_{\text {diff }}$ in 9 .
} 
that they are the consequence of a poor choice in setting up the details of the mathematical framework of the theory. It is not unusual that a naive way of setting up a QFT produces a nonseparable Hilbert space, which is later cured, making it separable. Indeed, recall that Fock space itself, the prototypical QFT state space, was born precisely to cure nonseparability. A free scalar field can be decomposed into an infinite set of oscillators $\varphi_{i}, i=1, \ldots, \infty$. If we quantize each degree of freedom $\varphi_{i}$ as a standard quantum harmonic oscillator, we are immediately led to a state space which is the tensor product of an infinite number of separable Hilbert spaces

$$
\mathcal{H}=\bigotimes_{i=1, \ldots, \infty} H_{i}
$$

A basis in $\mathcal{H}$ is given by an infinite sequence of nonnegative integers $\left|n_{1}, n_{2}, \ldots, n_{i}, \ldots,\right\rangle$, and such infinite sequences are noncountable (for instance, the states with $n_{i}<10$ are put into one-to-one correspondence with the real numbers $0 \leq x<1$ by the decimal representation of $x$ ); hence $\mathcal{H}$ is nonseparable. Fock found a way to circumvent the problem by simply selecting the subspace $\mathcal{F}$ of $\mathcal{H}$ spanned by basis vectors where an arbitrary but finite number of $n_{i}$ differ from zero. It is $\mathcal{F}$, called today Fock space, which is the appropriate state space for free QFT. Unlikely $\mathcal{H}$, the Fock space $\mathcal{F}$ provides an irreducible representation of the field algebra of the creation and annihilation operators. Thus, a straightforward and simple minded quantization strategy leading to a nonseparable state space has been later corrected to get rid of the nonseparability. Can we do the same in quantum gravity? Are there physical reasons for doing the same in quantum gravity?

In classical field theory, the choice of the functional class of fields and coordinates is to a large extent just a matter of convenience. We can work with solutions of the field equations that are smooth $\left(C^{\infty}\right)$, or just twice differentiable $\left(C^{2}\right)$, sometimes distributional, or piecewise constant as in the lattice approximation, or else, according to what is more convenient. The relation between the formal apparatus of field theory and reality is only via the smearing of fields on finite regions of space, and therefore we never empirically test the functional class of a physical field. In quantum gravity, the smooth category is generally chosen, because it appears to be a natural and convenient setting. In the background-independent loop construction, the gauge invariance of general relativity washes away virtually any remnant of the functional class we started from, because the entire short-scale structure is cancelled by the gauge transformations. Virtually, but not entirely. Indeed, as we show below, the nodes of sufficiently high valence have a surprising "rigidity" under smooth transformation, and this rigidity turns out to be the one responsible for the moduli. Therefore the non-separability of $\mathcal{H}_{\text {diff }}$ is a bizarre remnant of the initial choice of the smooth category. It is therefore natural to explore the possibility of using a slightly different functional class of fields to start with.

Here we explore a minimal extension of this kind, where fields are allowed isolated points of non-differentiability. The gauge invariance group of the theory becomes then a "small" extension of the diffeomorphism group, which we call extended diffeomorphism and denote Diff*. As far as we can see, none of the physical results, physical predictions or physical consequences of LQG studied so far, are affected. However, the knot classes, now formed by graphs under Diff*, turn out to be countable. The continuous moduli disappear and $\mathcal{H}_{\text {diff }}$ becomes separable. The theory describes a quantum structure of spacetime that is genuinely combinatorial at the Planck-scale.

These results can be taken as evidence that the moduli associated to high-valence nodes are indeed spurious. If we build LQG using the setting described here, or a variant of the same, the moduli are not anymore present, and the kinematical Hilbert space of the diffeomorphism invariant states of LQG is separable.

In Section 2 we review the basic mathematical setting of LQG and the origin of the nonseparability. This article is mathematically self-contained but for physical motivations and details see 9. In Section 3 we introduce our main tool, Diff*, and show that the equivalence classes of graphs under Diff* are countable and the resulting space $\mathcal{H}_{\text {diff }}$ is separable. In Section 4 , we show 
that the modification introduced has no effect on the geometrical operators of LQG. We discuss in particular the area operator. In Section 5 we summarize and conclude.

\section{Nonseparability}

\section{$2.1 \mathcal{H}_{\text {diff }}$, the space of the quantum-states of physical-space}

We begin by briefly reviewing the basic mathematical setting of the kinematics of LQG. Classical general relativity can be defined on a four-dimensional differentiable manifold $M$ with topology $\Sigma \times R$, where $\Sigma$ is a 3 -dimensional differentiable manifold with a fixed arbitrary topology. The gravitational field can be taken to be a smooth (that is, infinitely differentiable) pseudo-riemannian metric tensor $g$ on $M$, satisfying the Einstein equations. Call $\mathcal{E}$ the space of such fields. The corresponding hamiltonian theory can be defined on $\Sigma$. The configuration variable of general relativity can be defined as a smooth local connection one-form $A$ on a $S U(2)$-principal bundle $\mathcal{P}$ over $\Sigma$. Call $\mathcal{A}$ the space of such connections.

We shall use extensively the notion of graph, whose relevance for quantum gravity was first realized by Lewandowski. Here a graph $\Gamma$ is a finite collection of $L(\Gamma)$ smooth oriented onedimensional submanifolds of $\Sigma$, called links and noted $l$, overlapping (if they do) only at endpoints, called nodes and denoted $n$. We say that the $\operatorname{link} l$ ends at the node $n$ if $n$ is a boundary point of $l$. The valence of a node $n$ is the number of links ending at $n$. We call $\mathcal{G}$ the space of such graphs.

Quantum states are limit of sequences of cylindrical functions, converging in the norm defined below. A cylindrical function $\Psi_{\Gamma, f}: \mathcal{A} \rightarrow \mathbb{C}$ is defined as follows. Let $U_{l}(A) \in S U(2)$ be the holonomy of the connection $A$ along $l$

$$
U_{l}(A) \equiv \mathcal{P} \exp \int_{l} A
$$

$\mathcal{P}$ denotes path ordering. A graph $\Gamma$ defines a map $p_{\Gamma}: \mathcal{A} \rightarrow[S U(2)]^{\times L(\Gamma)} ; A \rightarrow\left(U_{l}(A)\right)$. By composing this map with a complex valued function $f$ on $[S U(2)]^{\times L(\Gamma)}$, we obtain the cylindrical function $\Psi_{\Gamma, f}=f \circ p_{\Gamma}$, given by

$$
\Psi_{\Gamma, f}(A) \equiv f\left(U_{l_{1}}(A), \ldots, U_{l_{L(\Gamma)}}(A)\right) .
$$

Since it is always possible to write any two cylindrical functions in terms of the same graph (a cylindrical function on a graph $\Gamma$ can be reexpressed on another graph $\Gamma^{\prime}$ that contains $\Gamma$ ), we can define a scalar product on the space of states using the Haar measure on $S U(2)$

$$
\left\langle\Psi_{(\Gamma, f)} \mid \Psi_{\left(\Gamma, f^{\prime}\right)}\right\rangle \equiv \int d U_{1} \ldots d U_{L(\Gamma)} \overline{f\left(U_{1}, \ldots, U_{L(\Gamma)}\right)} f^{\prime}\left(U_{1}, \ldots, U_{L(\Gamma)}\right) .
$$

The kinematical Hilbert space $\mathcal{K}$ of LQG is defined as the completion in the Hilbert norm (4) of the space of the cylindrical functions.

Local $S U(2)$ gauge transformations act naturally on this space and the invariant states form a proper subspace $\mathcal{K}_{0}$. An orthonormal basis in $\mathcal{K}_{0}$ can be obtained using the spin-network states [11, 3]. Consider a graph $\Gamma$ and color each link $l$ with a unitary irreducible representations $j_{l}$ of $S U(2)$. At each node $n$, fix a basis in the space of the invariant elements (the "intertwiners") in the tensor product of the representation spaces associated to the links that end at $n$, and color the node with an intertwiner $i_{n}$ of this basis (see 9] for details). The triple $S=\left(\Gamma, j_{l}, i_{n}\right)$ is called a spin network. Let the function $f_{S}$ be defined by the representation matrices for each link $l$ in the representation $j_{l}$, contracted with the invariant tensors at each node. The state $\Psi_{S}[A] \equiv \Psi_{\Gamma, f_{S}}[A] \equiv\langle A \mid S\rangle$ is a spin network state. Varying the graph $\Gamma$ in $\mathcal{G}$, the irreducible 
representations $j_{l}$ and the intertwiners $i_{n}$ in the chosen bases, we obtain an orthonormal basis in $\mathcal{K}_{0}$.

Finite linear combinations of spin-network states form a dense subspace $\mathcal{S}$ in $\mathcal{K}_{0}$. Sequences of states that converge weakly on $\mathcal{S}$, form the dual $\mathcal{S}^{\prime}$. The Gelfand triple $\left(\mathcal{S} \subset \mathcal{K}_{0} \subset \mathcal{S}^{\prime}\right)$ describes the space of the $S U(2)$ invariant states of the theory. Now, $\mathcal{K}_{0}$ carries a natural unitary representation $\Psi[A] \rightarrow U_{\phi} \Psi[A]=\Psi\left[\phi^{-1} A\right]$ of the group Diff Def $_{\Sigma}$ of diffeomorphisms of $\Sigma$

$$
\phi: \Sigma \rightarrow \Sigma
$$

which extends naturally to $\mathcal{S}^{\prime}$ by duality (we have indicated with $\phi$ also the pull back action generated by the diffeomorphism $\phi$ on forms). The diffeomorphism invariant states form a linear subspace $\mathcal{H}_{\text {diff }}$ of $\mathcal{S}^{\prime}$. This space describes the diff-invariant quantum states of the gravitational field; that is, the quantum states of physical space.

A map $P_{\text {diff }}: \mathcal{S} \rightarrow \mathcal{S}^{\prime}$ is naturally defined by

$$
\left(P_{\text {diff }} \Psi\right)\left(\Psi^{\prime}\right)=\sum_{\Psi^{\prime \prime}=U_{\phi} \Psi}\left\langle\Psi^{\prime \prime}, \Psi^{\prime}\right\rangle
$$

where the sum is over al distinct states $\Psi^{\prime \prime}$ that are equal to $U_{\phi} \Psi$ for some $\phi \in$ Diff. This sum converges and is well defined. The state $P_{\text {diff }} \Psi \in \mathcal{S}^{\prime}$ is diff-invariant because clearly states related by diffeomorphisms are projected by $P_{\text {diff }}$ on the same element of $\mathcal{H}_{\text {diff }}$

$$
P_{\text {diff }} \Psi_{S}=P_{\text {diff }}\left(U_{\phi} \Psi_{S}\right)
$$

Furthermore, the states of this form span $\mathcal{H}_{\text {diff }}$. The linear space $\mathcal{H}_{\text {diff }}$ is naturally equipped with a Hilbert space structure by the scalar product

$$
\left\langle P_{\text {diff }} \Psi_{S}, P_{\text {diff }} \Psi_{S^{\prime}}\right\rangle_{\mathcal{H}_{\text {diff }}} \equiv\left(P_{\text {diff }} \Psi_{S}\right)\left(\Psi_{S^{\prime}}\right)
$$

Equivalently, the Hilbert space $\mathcal{H}_{\text {diff }}$ can be defined by the bilinear form

$$
\left\langle\Psi, \Psi^{\prime}\right\rangle_{\mathcal{H}_{\text {diff }}} \equiv\left\langle\Psi\left|P_{\text {diff }}\right| \Psi^{\prime}\right\rangle \equiv \sum_{\Psi^{\prime \prime}=U_{\phi} \Psi}\left\langle\Psi^{\prime \prime}, \Psi^{\prime}\right\rangle .
$$

We can unravel the structure of $\mathcal{H}_{\text {diff }}$ by studying the action of a diffeomorphism on a spin network state. Since the holonomy transforms as

$$
U_{l}\left(\phi^{-1} A\right)=U_{\phi \circ l}(A)
$$

shifting $A$ with $\phi \in \operatorname{Diff}(\Sigma)$ is equivalent to shifting the curve $l$. Consequently, the (representation of the) spatial diffeomorphism group acts directly on the spin network of a spin-network state:

$$
U_{\phi}|S\rangle=|\phi \circ S\rangle
$$

Since $|\phi \circ S\rangle$ may be formed by invariant tensors that are not in the intertwiner basis chosen at the nodes, $|\phi \circ S\rangle$ may fail to be a basis state even if $|S\rangle$ is; but as the spaces of intertwiners are finite dimensional, it will be a finite linear combination of basis states, all having the same graph $\Gamma^{\prime}=\phi \circ \Gamma$. In particular, given a spin network state, there is a finite group of transformations $g_{k}$, $k=1, \ldots, K$, in the (tensor over the nodes of the) spaces of the intertwiners, that can be obtained by a diffeomorphism mapping the graph into itself. We can therefore write

$$
\left\langle S\left|P_{\text {diff }}\right| S^{\prime}\right\rangle=\left\{\begin{array}{cl}
0 & \text { if } \Gamma^{\prime} \neq \phi \circ \Gamma, \\
\sum_{k}\left\langle S\left|g_{k}\right| S^{\prime}\right\rangle & \text { if } \Gamma^{\prime}=\phi \circ \Gamma .
\end{array}\right.
$$


From the first line, we see that two spin networks $S$ and $S^{\prime}$ define orthogonal states in $\mathcal{H}_{\text {diff }}$ if the corresponding graphs $\Gamma$ and $\Gamma^{\prime}$ belong to different equivalence classes under diffeomorphism transformations. We call these equivalence classes "diff-knot classes" and indicate them as $K_{\mathrm{d}}$, where "d" is for diffeomorphism. The basis states in $\mathcal{H}_{\text {diff }}$ are therefore firstly labelled by a diff-knot class $K_{\mathrm{d}}$. Call $\mathcal{H}_{K_{\mathrm{d}}}$ the subspace of $\mathcal{H}_{\text {diff }}$ spanned by the basis states labelled by the knot class $K_{\mathrm{d}}$. The states in $\mathcal{H}_{K_{\mathrm{d}}}$ are then only distinguished by the coloring of links and nodes. Hence $\mathcal{H}_{K_{\mathrm{d}}}$ is separable. These colorings are however not necessarily orthogonal because of the nontrivial action of the discrete group. A diagonalization of the matrix of the bilinear form in the second line of expression (12) yields states $|s\rangle=\left|K_{\mathrm{d}}, c\right\rangle$, where the discrete label $c$ depends only on colorings. The states $|s\rangle=\left|K_{\mathrm{d}}, c\right\rangle$ are called $s$-knot states.

If $|s\rangle=P_{\text {diff }}|S\rangle$, the state $|s\rangle$ represents the diffeomorphism equivalence class to which the spin network $S$ belongs. In going from from the spin network state $|S\rangle$ to the $s$-knot state $|s\rangle$, we preserve the information in $|S\rangle$ except for it's location in $\Sigma$. This is the quantum analog to the fact that physically distinguishable solutions of the classical Einstein equations are not fields, but equivalence classes of fields under diffeomorphisms. It reflects the core of the conceptual revolution of general relativity: spatial localization concerns only the relative location of the dynamical fields, and not their location in a background space. Accordingly, the $s$-knot states are not quantum excitations in space, they are quantum excitation of space. An $s$-knot does not reside "somewhere": the $s$-knot itself defines the "where".

However, a remnant of the background structure oddly remains, when a node has sufficiently high valence, as we show in the next section.

\subsection{Moduli space structure}

We have seen above that quantum states of the gravitational field are labelled by diff-knot classes. Following [6], we now study the structure of these classes and the way this affects the structure of $\mathcal{H}_{\text {diff }}$. The key point that we underline below is the fact that diff-knots are not countable, hence $\mathcal{H}_{\text {diff }}$ is nonseparable.

To begin with, consider usual knots, namely ones without intersections. These can be defined in two equivalent manners. Let $\mathcal{L}$ be the space of loops without intersections, namely the space of smooth embeddings of $S_{1}$ into $\Sigma$. We denote loops in $\mathcal{L}$ as $\alpha, \beta, \ldots$. Consider two equivalence relations on this space. First (see for instance [5]) we say that $\alpha$ and $\beta$ are iso-equivalent, and write $\alpha \sim_{\mathrm{i}} \beta$, if there is a continuous ambient isotopy relating the two; that is, a one parameter family of homeomorphisms $h_{t}: \Sigma \rightarrow \Sigma, t \in[0,1]$, such that $h_{0}$ is the identity map on $\Sigma$ while $h_{1}$ maps $\alpha$ to $\beta$. We call the equivalence classes of loops in $\mathcal{L}$ under this equivalence relation "iso-knots". Next, (see for instance [2]) we say that $\alpha$ and $\beta$ are diff-equivalent, and write $\alpha \sim_{\mathrm{d}} \beta$ if there exists a diffeomorphism $\phi$ of $\Sigma$ in the connected component of the identity, such that $\alpha=\phi \circ \beta$ (or, equivalently, if there exists a smooth ambient isotopy relating the two). We denote the corresponding equivalence classes in $\mathcal{L}$ as "diff-knots". A classic result of knot theory states that two loops are diff-equivalent if and only if they are iso-equivalent. Hence diffeomorphism equivalence is the same as isotopy, as far as loops without intersections are concerned, and a diff-knot is also an iso-knot. ${ }^{3}$

However, this result does not extend to the case in which intersections, or nodes, are present; something peculiar happens at the intersection points. Let $\mathcal{G}$ be the space of the graphs defined in the previous section, of which $\mathcal{L}$ is a subset. Define isotopy equivalence and diff-equivalence between graphs precisely as we did for loops. Let's indicate iso-knots by $K_{\mathrm{i}}$ and the space of the iso-knots as $\mathcal{K}_{\mathrm{i}}$. Similarly, let's indicate diff-knots by $K_{\mathrm{d}}$ and the space of the diff-knots as $\mathcal{K}_{\mathrm{d}}$.

\footnotetext{
${ }^{3}$ This is also equivalent to the existence of a smooth homotopy between $\alpha$ and $\beta$, that is, a smooth one-parameter family of embeddings $\alpha_{t}, t \in[0,1]$, such that $\alpha_{0}=\alpha$ and $\alpha_{1}=\beta$. However, this is not equivalent to to the existence of a continuous homotopy between $\alpha$ and $\beta$, since, perhaps surprisingly, any two knots can be related by continuous homotopy. See for instance Ref 8 page 14 or Ref [5], exercise 3.5.4 page 53.
} 
Thus

$$
\mathcal{K}_{\mathrm{i}}=\frac{\mathcal{G}}{\sim_{\mathrm{i}}}, \quad \mathcal{K}_{\mathrm{d}}=\frac{\mathcal{G}}{\sim_{\mathrm{d}}} .
$$

It is still easy to see that diff-equivalence implies iso-equivalence, because a diffeomorphism in the connected component of the identity is indeed connected to the identity by a smooth one parameter family of diffeomorphisms which generates the isotopy. But now the converse is not true: there are iso-equivalent graphs that are not diff-equivalent. Furthermore, while iso-knots are countable, diff-knots are not: they are distinguished by moduli. (In general, a "modulus" is a continuous parameter labelling equivalence classes.)

The roots of this fact, which at first might seem surprising, can be illustrated using a simple example presented by Arnold in his book on catastrophe theory [1]. Let $L_{n}$ be the set of all $n$-tuples of lines in the plane, passing through the origin. Say that two $n$-tuples are equivalent if they can be mapped into each other by a linear transformation of the plane, and let $T_{n}$ be the space of the equivalence classes of $n$-tuples under this equivalence relation. The spaces $T_{1}, T_{2}$ and $T_{3}$ are discrete, but $T_{4}$ is a one-dimensional space. Indeed the group of linear transformation of the plane, $G L(2)$, is four dimensional, but it does not act effectively on $T_{n}$ because the dilatations do not affect lines through the origin, hence we can consider just its $S L(2)$ subgroup, which is three dimensional. But $L_{4}$ is four dimensional, and the dimension of $T_{4}=L_{4} / S L(2)$ is $4-3=1$. More explicitly, if $\phi_{1}, \ldots, \phi_{4}$ are the angles formed by the lines with a given axis, then it is easy to see that

$$
\lambda\left(\phi_{1}, \ldots, \phi_{4}\right)=\frac{\sin \left(\phi_{1}-\phi_{3}\right) \sin \left(\phi_{2}-\phi_{4}\right)}{\sin \left(\phi_{1}-\phi_{4}\right) \sin \left(\phi_{2}-\phi_{3}\right)}
$$

is invariant under linear transformations. Indeed, let $\vec{v}_{1}, \ldots, \vec{v}_{4}$ be four vectors of arbitrary length parallel to the four lines. The ratio

$$
\lambda\left(\vec{v}_{1}, \ldots, \vec{v}_{4}\right)=\frac{\left(\vec{v}_{1} \times \vec{v}_{3}\right)\left(\vec{v}_{2} \times \vec{v}_{4}\right)}{\left(\vec{v}_{1} \times \vec{v}_{4}\right)\left(\vec{v}_{2} \times \vec{v}_{3}\right)},
$$

where $\vec{v}_{1} \times \vec{v}_{2}=\operatorname{det}\left(\vec{v}_{1}, \vec{v}_{2}\right)$ is the $2 \mathrm{~d}$ vector product, is invariant under linear transformations because the vector product transforms with the determinant of the linear transformation. On the other hand, the ratio does not depend on the length of the vectors, and is equal to (14). Therefore $\lambda$ is a continuous modulus that distinguishes $G L(2)$ equivalence classes in $L_{4}$, and $T_{4}$ is a continuous space.

The same happens in three dimensions. Here $G L(3)$ has nine dimensions, of which only eight affect the $n$-tuplets of lines through the origin; five lines through the origin are determined by ten angles; hence in $3 \mathrm{~d} T_{n}=L_{n} / G L(3)$ is only discrete for $n<5$. If $\vec{v}_{1}, \ldots, \vec{v}_{5}$ are five vectors of arbitrary length parallel to the five lines, the ratio

$$
\lambda\left(\vec{v}_{1}, \ldots, \vec{v}_{5}\right)=\frac{\vec{v}_{1} \cdot\left(\vec{v}_{2} \times \vec{v}_{3}\right)}{\vec{v}_{1} \cdot\left(\vec{v}_{4} \times \vec{v}_{5}\right)},
$$

where $\vec{v}_{1} \cdot\left(\vec{v}_{2} \times \vec{v}_{3}\right)=\operatorname{det}\left(\vec{v}_{1}, \vec{v}_{2}, \vec{v}_{3}\right)$ is the triple product, is invariant under linear transformation. It classifies quintuplets of straight lines into equivalence classes. The invariant (15) is well known in projective geometry since the nineteenth century, as the cross-ratio. It distinguishes orbits of quadruplets of points generated by the action of the projective group on the real projective line. The generalization of the cross-ratio projective invariant to higher dimensions has been studied by Hilbert.

Let us return to nodes. Consider for simplicity a graph $\Gamma$ with a single node $n$. Say that the node is five-valent. Let $\Gamma^{\prime}$ be a node iso-equivalent to $\Gamma$. The graph $\Gamma^{\prime}$ will have a single five-valent node $n^{\prime}$ as well. Say, for simplicity, that $n^{\prime}$ is located at the same point $p$ as $n$. Can we always find a diffeomorphism $\phi$ that sends $\Gamma^{\prime}$ into $\Gamma$ ? A condition on $\phi$ is that $\phi(p)=p$. The tangent map $\phi_{*}: T_{p} \rightarrow T_{p}$ defines a linear action on the tangent space $T_{p}$ of $\Sigma$ at $p$. The tangents to the five 
links of $\Gamma$ at $p$ determine a quintuplet of straight lines in $T_{p}$. Similarly, the tangents to the links of $\Gamma^{\prime}$ determine another quintuplet of straight lines in $T_{p}$. A condition on $\phi$ is then that the linear transformation $\phi_{*}$ maps the first quintuplet of lines into the second. As observed above, in general such a linear transformation does not exist. Equivalence classes under linear transformations of quintuplets of lines are distinguished by continuous moduli. If $\vec{v}_{i}(\Gamma, n), i=1, \ldots, 5$ are the tangents to the five links of $\Gamma$ at the node $n$, in an arbitrary parametrization and an arbitrary coordinate system, the quantity

$$
\lambda(\Gamma, n)=\lambda\left(\vec{v}_{1}(\Gamma, n), \ldots, \vec{v}_{5}(\Gamma, n)\right),
$$

where the function on the right hand side is defined in (16), does not depend neither on the parametrization nor on the coordinates chosen, and is invariant under diffeomorphisms. Hence if $\lambda(\Gamma, n) \neq \lambda\left(\Gamma^{\prime}, n^{\prime}\right)$, the two iso-equivalent graphs $\Gamma$ and $\Gamma^{\prime}$ are not diff-equivalent. The function (17) is an example of the continuous moduli that distinguish diff-knots.

In general, a single iso-knot $K_{\mathrm{i}}$ is therefore formed by a continuous set of diff-knots $K_{\mathrm{d}}$; diffknots are labelled by moduli at the intersections. These moduli have a structure far richer than Hilbert's projective invariants. Indeed, it is not only the tangent structure that may distinguish iso-equivalent nodes, but also, for sufficiently high valence, the higher derivatives of the links at the intersection. For instance, assume that the tangents of the links at the node of two graphs are the same, but their curvatures differ. Under a diffeomorphism $\phi$, the transformation of these curvatures is determined by the second derivatives of $\phi$ at the node, but these are finite in number as well (they are 18) and therefore for a sufficiently high valence they are not sufficient, in general, to map all curvatures of one graph into the curvatures of the second. We refer to [6] for a detailed analysis of the structure of the moduli.

We have seen in the previous section that the diff-knots $K_{\mathrm{d}}$ label a basis in $\mathcal{H}_{\text {diff }}$. Since they are not countable, the kinematical state space $\mathcal{H}_{\text {diff }}$ space admits a continuous basis and therefore is nonseparable. Thus the root of the nonseparability of the state space of LQG is the "rigidity" of the diffeomorphisms at isolated points: the differential structure of the underlying manifold is rigid in the sense that it produces the linear structure of the tangent spaces $T_{p}$, which leaves quantities such as the cross-ratio (14) invariant.

\subsection{Discussion}

The nonseparability of the kinematical Hilbert space $\mathcal{H}_{\text {diff }}$ is disturbing for several reasons.

First, the background independence of general relativity implies that the localization of the dynamical fields on the coordinate manifold has no physical meaning: only relative localization of dynamical objects with respect to one another is physically significant. In the classical theory, background independence is implemented by the fact that diffeomorphisms turn out to be gauges. When implementing gauge invariance in the quantum theory, the localization of the spin network in the manifold is washed away by the gauge transformation, and only the discrete combinatorial relations remain - but not completely so. The moduli distinguishing diff-knots are a remnant of the localization of the spin network in the coordinate manifold. It is difficult to reconcile the presence of this remnant with the physical principle, underlying general relativity, that wants the localization on the coordinate manifold to be physically irrelevant.

Second, recall that loop states and spin-network states form a good basis in lattice Yang-Mills theory, but in continuous Yang-Mills theory they are "too singular" and "too many", because of their continuous dependence on position. In gravity, the continuous dependence of position is gauged away by diffeomorphism invariance, dramatically reducing the size of the resulting state space [10, 12. This is the reason for which the loop basis becomes viable thanks to background independence, and therefore the rationale underlying the background-independent loop quantization. It is quite puzzling that this dramatic reduction of the state space fails to be complete because of the moduli. 
Third, if we accept the formalization of generally covariant quantum theory described in [9], the Hilbert space $\mathcal{H}_{\text {diff }}$ describes distinguishable quantum states. A realistic space of distinguishable quantum states should be described by a separable Hilbert space [13.

Now, if the continuous moduli had a physical meaning, they should affect measurements, or affect the dynamics. Two states that differ only by different values of their moduli should in principle be distinguishable by means of physical measurements. However, as mentioned in the introduction, this does not appear to be the case. The only effect of the moduli appears to be to vastly enlarging the kinematical Hilbert space, with no visible effect on the physics of the theory. One is therefore naturally led to the idea that these moduli may be spurious.

Above we have observed that the moduli are a consequence of the incapacity of diffeomorphisms to act at vertices in a way sufficiently general to map iso-equivalent graphs into each other. Diffeomorphisms are "rigid" at vertices. If gauge transformations included maps $\phi: \Sigma \rightarrow \Sigma$ less rigid at nodes (homeomorphisms, for instance), the continuous moduli would disappear: states distinguished by different moduli would become gauge equivalent. So, it is natural to ask: what is it that forces gauge transformations $\phi: \Sigma \rightarrow \Sigma$ implementing background-independence, to be diffeomorphisms? Namely to be smooth?

To a large extent, the answer is: just the conventional way we set up the theory. It is simple and convenient to use smooth fields on a smooth manifold, and stay in the smooth category. The moduli are a consequence of this choice, which might have little do to with physics. It is therefore natural to investigate the possibility of a different mathematical starting point, that would not affect classical physics, but would free the quantum theory from the moduli.

A very natural setting one may consider is the piecewise smooth category. Another possibility, investigated by Josè Zapata [15] is to start from a piecewise linear manifold. With these choices, the moduli disappear and $\mathcal{H}_{\text {diff }}$ becomes separable. In this paper we investigate a minimal choice: we consider fields that are everywhere continuous and smooth everywhere except possibly at a finite number of points. We call these fields "almost smooth". This minor enlargement of the space of the fields has practically no effect on the classical theory, nor on the physical results of LQG. But it gets rid of moduli and nonseparability.

\section{Extended diffeomorphism group}

We now define a modified theory, where the gauge group is an extension of the diffeomorphism group. We show that the knot classes defined by the equivalence relation determined by this extended gauge group are countable, and lead to a separable $\mathcal{H}_{\text {diff }}$.

\subsection{Almost smooth physical fields}

Consider a four-dimensional differentiable manifold $M$ with topology $\Sigma \times R$, as before. However, we now allow the gravitational field $g$ to be almost smooth, as defined in the previous section, that is: $g$ is a continuous field which is smooth everywhere except possibly at a finite number of points, which we call the singular points of $g$. Any such $g$ can be seen as a (pointwise) limit of a sequence of smooth fields. We say that $g$ is a solution of the Einstein equations if it is the limit of a sequence of a sequence of smooth solutions of the Einstein equations. Call $\mathcal{E}^{*}$ the space of such fields.

Let now $\phi$ be an invertible map from $M$ to $M$ such that $\phi$ and $\phi^{-1}$ are continuous and are infinitely differentiable everywhere except possibly at a finite number of points. The space of these maps form a group under composition, because the composition of two homeomorphisms that are smooth except at a finite number of singular points is clearly an homeomorphisms which is smooth except at a finite number of singular points. We call this group the extended diffeomorphism group 


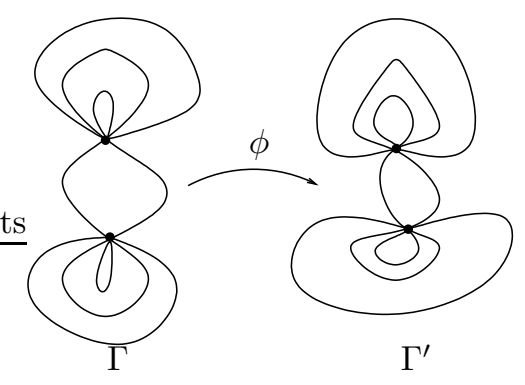

Figure 1: Two graphs living in the same iso-knot but not in the same diff-knot.

and we denote it as Diff ${ }_{M}^{*}$. It is clear that if $g \in \mathcal{E}^{*}$ then $(\phi g) \in \mathcal{E}^{*}$ for any $\phi \in \operatorname{Diff}_{M}^{*}$. Hence $D_{i f f}^{*}$ is a gauge group for the theory.

In the Hamiltonian theory, we can now take almost smooth connections $A$ on $\Sigma$. Notice that the holonomy of an almost smooth connection on a link $l$ is well defined, because it is the product of the holonomies on the portions in which $l$ is partitioned by the eventual singular points of $A$. We can thus define cylindrical functions, $\mathcal{K}$ and $\mathcal{K}_{0}$ as before. However, the gauge group Diff is now replaced by the gauge group Diff* ${ }^{*}$, formed by the homeomorphisms of $\Sigma$ that are almost smooth (with their inverse). The group Diff considered above is a dense subgroup of Diff* 4 . Notice that Diff* has a well defined action on the space of the graphs $\mathcal{G}$. Unlike Diff, Diff * does not preserve the number of nodes of a graph, because a singular point of $\phi$ may break a link into two links and create a bivalent node.

The construction $\mathcal{H}_{\text {diff }}$ is the same as before, with the only difference that $\phi$ in (12) is now in Diff* . Hence $\mathcal{H}_{\text {diff }}$ is now spanned by a basis of states $|s\rangle=\left|K_{\mathrm{d}^{*}}, c\right\rangle$ where $c$ is a discrete quantum number as before, but $K_{\mathrm{d}^{*}}$ is an element of $\mathcal{K}_{\mathrm{d}^{*}}$, namely an equivalence class of graphs under extended diffeomorphisms

$$
\mathcal{K}_{\mathrm{d}^{*}}=\frac{\mathcal{G}}{\sim_{\mathrm{d}^{*}}}
$$

where $\Gamma \sim_{\mathrm{d}^{*}} \Gamma^{\prime}$ if there is a $\phi \in D i f f *$ such that $\Gamma^{\prime}=\phi \circ \Gamma$. We denote the elements $K_{\mathrm{d}^{*}}$ of $\mathcal{K}_{\mathrm{d}^{*}}$ as diff*-knots.

\subsection{Diff*-knots are countable}

We now prove that diff*-knots are countable. Two diff-equivalent graphs are also diff*-equivalent, because Diff is a subgroup of Diff*. Therefore diff*-knots are equivalence classes of diff-knots. To prove that diff*-knots are countable it is sufficient to prove that any two diff-knots distinguished by a continuous parameter are diff*-equivalent. For this, it is sufficient to consider two isoequivalent but diff-inequivalent graphs $\Gamma$ and $\Gamma^{\prime}$. Our strategy will be to explicitly build an extended diffeomorphism mapping $\Gamma$ into $\Gamma^{\prime}$ (see Figure 1). Since iso-knots are countable, this will be sufficient to show that $\mathcal{K}_{d^{*}}$ is countable.

Choose an arbitrary smooth metric $g$ on $\Sigma$. Consider a node $n$ of $\Gamma$, say with valence $v$. Consider the open ball $B_{n}$ of fixed radius $\epsilon(n)$ about $n$. Let $S_{r}$ be the spheres of radius $r(n)$, $0<r(n)<\epsilon(n)$, centered at the same point $n$. Introduce (nonmetrical) angular coordinates $(\theta, \phi)_{r}$ on each sphere $S_{r}$ of radius $r$, smooth in $r$. By choosing $\epsilon$ sufficiently small, each link $l$ ending at $n$ will intersect each sphere only once, say in the point with coordinates $\left(\theta_{l}, \phi_{l}\right)_{r}$. (No fold-backs, see Figure 2.) Now, we can always choose the coordinates $(\theta, \phi)_{r}$ in such a way that the coordinates

${ }^{4}$ Diff* $^{*}$ can be given a topological group structure as a subgroup of the homeomorphism group of $\Sigma$. The question of whether it can be given a Lie group structure is more difficult. 
$\left(\theta_{l}, \phi_{l}\right)_{r}$ of the link $l$ are independent from $r$, and are equal to $v$ arbitrarily chosen values $\left(\theta_{l}, \phi_{l}\right)$. This construction can be repeated for the node $n^{\prime}$ of $\Gamma^{\prime}$ which is isotopically associated to the node $n$. For the coordinates on the ball $B_{n^{\prime}}$ around the node $n^{\prime}$ we use the notation $\left(r^{\prime}, \theta^{\prime}, \phi^{\prime}\right)$ and we choose $\left(\theta_{l^{\prime}}^{\prime}=\theta_{l}, \phi_{l^{\prime}}^{\prime}=\phi_{l}\right)$, namely equal angular coordinates for corresponding links. We call $\Gamma_{n}$ the restriction of $\Gamma$ to $B_{n}$ and $\Gamma_{n}^{\prime}$ the restriction of $\Gamma^{\prime}$ to $B_{n^{\prime}}$.

Consider now the space $\Sigma \backslash B_{n}$ obtained by removing all balls $B_{n}$, for all nodes $n$, from $\Sigma$, and the space $\Sigma \backslash B_{n}^{\prime}$ obtained removing all balls $B_{n^{\prime}}$. Let $\tilde{\Gamma}$ be the restriction of $\Gamma$ to $\Sigma \backslash B_{n}$ and $\tilde{\Gamma}^{\prime}$ be the restriction of $\Gamma^{\prime}$ to $\Sigma \backslash B_{n^{\prime}}$. Under the hypothesis we made that $\Gamma$ and $\Gamma^{\prime}$ are iso-equivalent, a smooth invertible map

$$
\begin{array}{ccc}
\tilde{\phi}: \Sigma \backslash B_{n} & \rightarrow & \Sigma \backslash B_{n}^{\prime}, \\
\tilde{\Gamma} & \mapsto & \tilde{\Gamma}^{\prime}
\end{array}
$$

exists, because we are here in the simpler case of loops without intersections (on a space with boundaries), where standard knot-theory results apply. The failure of iso-equivalence to yield diffeo-equivalence regards only the neighborhoods of the nodes.

To prove that $\Gamma$ and $\Gamma^{\prime}$ are diff*-equivalent, we have therefore just to construct maps

$$
\begin{aligned}
\phi_{n}: B_{n} & \rightarrow B_{n}^{\prime}, \\
\Gamma_{n} & \mapsto \Gamma_{n}^{\prime}
\end{aligned}
$$

such that $\tilde{\phi}$ and $\phi_{n}$, taken together, give an almost smooth map $\phi: \Sigma \rightarrow \Sigma$. Let $\phi_{n}$ be given simply by

$$
\begin{aligned}
\phi_{n}: B_{n} & \rightarrow B_{n}^{\prime}, \\
(r, \theta, \phi) & \mapsto \quad\left(r^{\prime}=r, \theta^{\prime}=\theta, \phi^{\prime}=\phi\right) .
\end{aligned}
$$

$\tilde{\phi}$ can be chosen so that at the boundaries of the balls $\phi$ is smooth. Hence $\phi$ is smooth for all $r>0$. The map $\phi_{n}$ can immediately be continued to $r=0$, yielding by continuity $\phi_{n}(n)=n^{\prime}$. But there is no reason for this continuation to be smooth, and in fact, in general it will not be. Hence $\phi$ is not in Diff. But it is in Diff*, because it is continuous, invertible and smooth everywhere except at the nodes, which are finite in number. Therefore iso-equivalent graphs $\Gamma$ and $\Gamma^{\prime}$ are diff*-equivalent. Therefore

Proposition 1 The space of the diff ${ }^{*}-k n o t s \mathcal{K}_{d^{*}}$ is countable.

It follows immediately that

Proposition 2 If $\phi \in$ Diff ${ }^{*}$, the space $\mathcal{H}_{\mathrm{diff}}$ defined by the bilinear form (12) is separable.

Therefore we have shown that a minor extension of the functional space of the fields considered eliminates the continuous moduli and the nonseparability of the kinematical state space of LQG.

\section{Operators}

In this section we discuss some consequences of the extension of Diff to Diff ${ }^{*}$ and clarify some apparent difficulties that this extension raises.

\subsection{Conical singularities and area operator}

If the gauge group is Diff*, a smooth 2-dimensional surface is gauge equivalent to a "singular" surface, that is a surface with conical singularities. The area operator $A(\mathcal{S})$ of LQG has been 


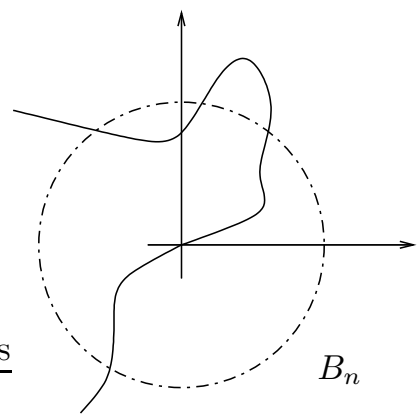

Figure 2: A Link presenting fold-backs.

defined for smooth surfaces $\mathcal{S}$. Is it well defined also for a singular surface $\mathcal{S}$ ? Naively, one may think that $A(\mathcal{S})$ is ill defined for a singular $\mathcal{S}$, for the following reason. Consider a two-dimensional surface $\mathcal{S}$ embedded in $\Sigma$. Let $x=\left(x^{a}\right), a=1,2,3$ be coordinates on $\Sigma$ and $u=\left(u^{m}\right), m=1,2$ coordinates on $\mathcal{S}$; the embedding is given by the functions $x^{a}(u)$. Let $g_{a b}(x)$ be the $3 \mathrm{~d}$ metric, namely the gravitational field. The classical expression for the area of $\mathcal{S}$ is

$$
A(\mathcal{S})=\int_{\mathcal{S}} d^{2} u \sqrt{\operatorname{det} g_{m n}(u)}
$$

where

$$
g_{m n}(u)=\frac{\partial x^{a}(u)}{\partial u^{m}} \frac{\partial x^{b}(u)}{\partial u^{n}} g_{a b}(x(u))
$$

is the two-dimensional metric induced on the surface. The area operator is constructed by expressing the area in terms of the variable canonically conjugated to $A$, which is the inverse densitized triad $E_{i}^{a}(x), i=1,2,3$, related to the metric by $\operatorname{det} q q^{a b}=E_{i}^{a}(x) E_{j}^{b}(x) \delta^{i j}$. This gives

$$
A(\mathcal{S})=\int_{A} d^{2} u \sqrt{n_{a} n_{b} E_{i}^{a} E_{j}^{b} \delta^{i j}}
$$

where

$$
n_{a}(u)=\epsilon_{a b c} \frac{\partial x^{b}(u)}{\partial u^{1}} \frac{\partial x^{c}(u)}{\partial u^{2}} .
$$

is the one-form normal to the surface $\mathcal{S}$. If $\mathcal{S}$ is singular at a point $p$, the normal $n_{a}(u)$ is not defined at $p$. This has no effect on the expression of the classical area (24) because the singular point is a set of measure zero. Obviously, indeed, the area of a cone is defined in the same manner as the area of a smooth surface. However, what happens at single points becomes important for the LQG quantum operator $A(\mathcal{S})$ that corresponds to the classical quantity (24). A spin network state determined by a spin network $S$ that crosses the surface at a single point $p$ contributes to the area of the surface. In the derivation of this contribution, the tangent to the link of $S$ at $p$ gets contracted with the normal $n_{a}(p)$. If this is ill defined, we might expect a problem.

The proper way of addressing this issue is in the context of a quantization of the area operator based on a well-defined regularization. Several equivalent regularization schemes to define area operator are discussed in the literature. Not all of these schemes can be immediately adapted to a surface with conical singularities, but the regularization discussed in 4, which uses a smearing transversal to the surface, remains well-defined for singular surfaces. This regularization is based on a continuous family of surfaces $\mathcal{S}_{\lambda}$, with $\lambda \in[-\delta / 2, \delta / 2]$, where $\delta$ is a positive real number, 


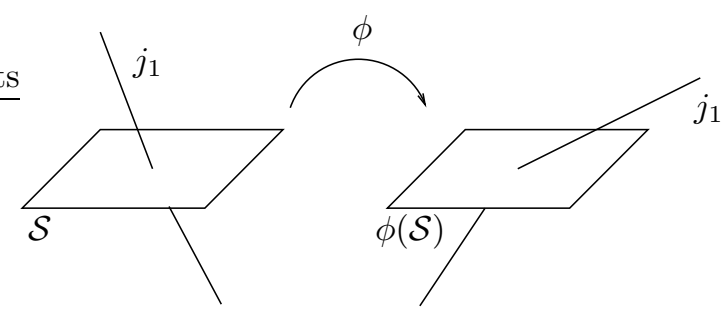

Figure 3: An extended diffeomorphism may generate a conical singularity but does not change the topological relation between a surface $\mathcal{S}$ and the link of a spin network.

such that $\mathcal{S}_{0}=\mathcal{S}$. To extend the technique to singular surfaces, we demand that $\mathcal{S}_{\lambda}$ is a smooth surface for $\lambda \neq 0$ and that $\mathcal{S}_{0}=\mathcal{S}$ is singular. The area of $\mathcal{S}$ is then written as the limit

$$
A(\mathcal{S})=\lim _{\delta \rightarrow 0} \frac{1}{\delta} \int_{-\delta / 2}^{\delta / 2} d \lambda A\left(\mathcal{S}_{\lambda}\right)=\lim _{\delta \rightarrow 0} \frac{1}{\delta} \int_{\mathcal{D}} d^{3} \sigma \sqrt{n_{a} n_{b} E^{a j} E_{j}^{b}}
$$

where $\mathcal{D}=\mathcal{S} \times[-\delta / 2, \delta / 2]$. The non vanishing contribution of the last integral comes now from the entire one-dimensional intersection between the spin network and the three dimensional region $\mathcal{D}$. In this, the contribution of the singular points of the $\lambda=0$ surface have measure zero. The fact that $\mathcal{S}$ has singular points is therefore irrelevant, and the operator $A(\mathcal{S})$ is well defined also for singular surfaces.

Recall that the LQG operator $A(\mathcal{S})$ is Diff-covariant in the sense that

$$
A(\phi(\mathcal{S}))=U_{\phi} A(\mathcal{S}) U_{\phi^{-1}}
$$

for all $\phi \in$ Diff. The above construction implies immediately that (27) remains true also if $\phi \in$ Diff $^{*}$, because the differentiable structure at the intersection point plays no role in the definition of $A(\mathcal{S})$. The action of the area operator of a singular surface $\mathcal{S}$ is therefore immediately obtained by (27) by choosing $\phi$ such that $\phi(\mathcal{S})$ is smooth. We see that what matters is not the linear structure at the intersection point, but just the topological relation between the surface and the spin networks defining the quantum states.

From this, it follows immediately that

Proposition 3 The spectrum of the operator $A(\mathcal{S})$ where $\mathcal{S}$ is singular (has a finite number of conical singularities) is the same as the spectrum of the operator $A(\mathcal{S})$ where $\mathcal{S}$ is smooth.

In conclusion, extended diffeomorphisms are indistinguishable from ordinary diffeomorphisms as far as the area operator is concerned. An extended diffeomorphism may generate singular points in the surface or in the spin network, but does not affect the topological relation between a surface and the spin network, and the area depends only on this relation.

\subsection{Volume and hamiltonian}

Call $V(\mathcal{R})$ the volume of a $3 \mathrm{~d}$ region $\mathcal{R}$ in $\Sigma$. There exist two versions of the volume operator $V(\mathcal{R})$ in LQG [7]. The first (let us call it $V_{1}(\mathcal{R})$ here), used for instance in 9], depends only on the intertwiners of the nodes inside $\mathcal{R}$. The second (let us call it $V_{2}(\mathcal{R})$ here), used for instance in 14, depends also on whether or not the links at the nodes are linearly dependent. The operator $V_{1}(\mathcal{R})$ is Diff*-covariant, that is

$$
V_{1}(\phi(\mathcal{R}))=U_{\phi} V_{1}(\mathcal{R}) U_{\phi^{-1}}
$$


for all $\phi \in$ Diff* $^{*}$ The operator $V_{2}(\mathcal{R})$, on the other hand, does not transform well under $\phi \in$ Diff*, because an extended diffeomorphism can modify the linear dependence of the links at the node. Therefore the formulation of LQG considered here requires the use of the version $V_{1}(\mathcal{R})$ of the volume operator.

Finally, the hamiltonian can be defined entirely in terms of the volume operator and holonomy operators, and is not affected by the modification of the theory considered here.

\section{Conclusion}

We have studied the problem of the separability of the background-independent space of the quantum states of the gravitational field, $\mathcal{H}_{\text {diff }}$, in loop quantum gravity. We have shown that a small extension of the functional class of the classical fields leads to an enlargement of the gauge group of the theory. In particular Diff is enlarged to Diff*, the group of homeomorphisms that are smooth (with their inverse) except possibly at a finite number of points. The space of the knot classes become countable and the kinematical Hilbert space $\mathcal{H}_{\text {diff }}$ is separable. The area, volume, and hamiltonian operator are naturally covariant under this extended gauge invariance, provided that the appropriate regularization and the appropriate version of the volume operator are chosen. The spectra of area and volume, in particular, are unaffected. We expect that analogous results could be obtained also using other mathematical settings, in particular the piecewise smooth category.

We take these results as indications that the continuous moduli that made $\mathcal{H}_{\text {diff }}$ nonseparable might be physically spurious. Using the setting described in this paper, the theory appears to be cleaner and to realize more completely its purely combinatorial character as well as background independence. If we adopt this point of view, background independent quantum microphysics is entirely discrete and smoothness can be seen, a posteriori, just as a property arising from averaging over regions much larger than the Planck scale.

\section{Acknowledgments}

Thanks to Philippe Roche for discussions and a careful reading of the paper, to Alain Connes for captivating remarks on the cross-ratio and its unfoldings and to Dan Christensen for clarification on knot theory and for pointing out an imprecision in the first draft of this paper.

\section{Note added in proofs}

After the posting of this work in the Archives, J. Lewandowski has informed us that related ideas have been developed by him and A. Ashtekar in work which is still unpublished.

\section{References}

[1] V.I. Arnold, Catastrophe Theory (Springer-Verlag, Berlin, 1984).

[2] J.C. Baez, J. Muniain, Gauge Fields, Knots and Gravity (World Scientific, 1994).

[3] J.C. Baez, "Spin networks in nonperturbative quantum gravity", in Interface of Knot Theory and Physics, L. Kauffman editor (American Mathematical Society, Providence, Rhode Island, 1996)

[4] S. Frittelli, L. Lehner and C. Rovelli, "The complete spectrum of the area from recoupling theory in loop quantum gravity", Class. Quant. Grav., 13:2921-2932, 1996. 
[5] N.D. Gilbert and T. Porter, Knots and Surfaces (Oxford Univiversity Press, Oxford 1994).

[6] N. Grot and C. Rovelli, "Moduli-space structure of knots with intersections", J. Math. Phys., 37:3014-3021, 1996.

[7] J. Lewandowski, "Volume and quantizations", Class. and Quantum. Grav. 14:71-76, 1997.

[8] C. Livingston, Knot Theory (The Mathematical Association of America, Washington 1993).

[9] C. Rovelli, Quantum Gravity (Cambridge University Press, Cambridge, 2004) to appear; a draft of the book can be found in http://www.cpt.univ-mrs.fr/ $\sim$ rovelli .

[10] C. Rovelli and L. Smolin: "Knot theory and quantum gravity", Phys. Rev. Lett. 61:1155, 1988; "Loop space representation for quantum general relativity", Nucl. Phys. B331:80, 1990.

[11] C. Rovelli and L. Smolin: "Spin networks and quantum gravity", Phys. Rev. D52:5743-5759, 1995.

[12] C. Rovelli and L. Smolin,: "Discreteness of Area and Volume in Quantum Gravity", Nucl. Phys. B442:593, 1995. Erratum: Nucl. Phys. B456:734, 1995.

[13] R.F. Streater and A.S. Wightman, PCT, Spin, Statistics, and all that (Benjamin, New York, 1964).

[14] T. Thiemann, Modern Canonical Quantum General Relativity (Cambridge University Press, Cambridge, to appear in 2004), a preliminary version is in gr-qc/0110034

[15] J. Zapata, "A combinatorial approach to diffeomorphism invariant quantum gauge theories", J. Math. Phys. 38:5663-5681, (1997); "A combinatorial space for loop quantum gravity", Gen. Rel. Grav. 30:1229, 1998. 